\title{
MIRCE Science Question: Digital or Analogue Visual Displays of Dynamic Information for Humans?
}

\author{
Jezdimir Knezevic* \\ MIRCE Akademy, Exeter, UK
}

*Corresponding author: Jezdimir Knezevic, MIRCE Akademy, Exeter, UK.

\begin{abstract}
Visual Displays of Dynamic Information (VDDI) represent the interface that machines use to communicate their dynamic functionability state to humans. This paper tries to address the question of the selection between the two main types of VDDI available, digital or analogue, and identify what some of the most important factors are that may affect this selection. In that aid the paper briefly covers how the displays and VDDI are classified, their uses and objectives, the factors that could affect the selection process and finally a comparison between digital and analogue VDDI. The quantitative assessment of the impact of VDDI on the human elements of functionable systems could be determined by making use of MIRCE Functionability Equation. It enables to predict the impact of each feasible options of VDDI on the expected work to be delivered by the system
\end{abstract}

\section{Introduction}

In system engineering the term human factors engineering is used to designate a body of knowledge related o the collection of data and principles about human characteristics, capabilities, and limitations in relation to machines, jobs, and environments taking account the safety, comfort, and productiveness of human users and operators. The term human factors engineering is used mainly in the North American continent. In Europe, Japan, and most of the rest of the world the prevalent term is ergonomics, a word formed from the Greek words, ergon, meaning "work," and nomos, meaning "law." Despite minor differences in emphasis, the terms human factors engineering, and ergonomics may be considered synonymous. The terms were used in the 1920 s and ' 30 s to refer to problems of human relations in industry, an older connotation that has gradually dropped out of use. Some specialized groups prefer such labels as bioastronautics, biodynamics, bioengineering, and manned-systems technology; these represent special emphases whose differences are much smaller than the similarities in their aims and goals.
The main problem with the system engineering approach to human factor is that operators, maintainers, and users are considered as an independent part of a system. For example, no human is a part of reliability block diagram, despite numerous evidences that humans had caused major accidents and failures. Hence, pilots, drivers, operators, maintainers, and other humans that actively participate in the life of systems are not considered as an integral part. To deal with this anomaly, and a few others, deeply imbedded in system engineering, MIRCE Science, has been created as an independent body of knowledge that studies and predicts the behavior of functionable systems. According to Knezevic [1], functionable system is "a set of mutually related entities put together to do a functionability work in accordance to physical laws and given functionability rules." The work is considered done when a measurable function is delivered through time. As, humans play different part in the working of systems, they are considered and an integral part of functionable system, and as such are included in the functionability block diagram of functionable systems. 
For example, a motor car is created to do a transporting work of people and cargo from one point to another, with expected speed, fuel consumption and other measurable performance. A refinery is built to do a work consisting of transforming crude oil into a variety of refined products as petrol, jet fuel, asphalt, oil, sulphur, etc. When these systems are performing their expected functions, humans participating in their operation need information about the functionability states of its consisting elements, to plan and execute all actions required to maintain the system in working state.

One of the many ways that systems use to communicate their functionability state to the humans, whether the system is a radio, a car, industrial plan or spaceship, is through visual displays. They provide the mechanism for the flows of information from noncognitive to cognitive elements of a functionable system. The visual displays enable humans to monitor the parameters that are direct indication of the functionability performance of the system.

According to James [2] the basic purpose of all displays is to provide answers to questions. For example, in the case of a car, the speed gauge informs the drivers about the speed, at any moment which they use to control the vehicle speed within the required limits, or the fuel gauge enables drivers to determine when to refuel. In the case of the refinery the manometer at the discharge of certain pump or the thermocouple at the outlet side of a heat exchanger will give critical information about how these devices are functioning, enabling humans to control or take corrective measurements in the event of a problem. The generic name for all equipment used to monitor parameters subject to change with time and inform humans about the functionability state of functionable systems is the Visual Displays of Dynamic Information (VDDI).

Given that the VDDI play a very important role in working of functionable systems the important question arises: "What method should be used to present visual information to humans in order that they are adequately, easily and timely understood, analogue or digital? Hence, the main purpose of this paper is to attempt to provide the answer the question raised, in accordance to MIRCE Science.

\section{Overview of Visual Displays of Dynamic Information}

Generally speaking, visual displays to humans take two forms: digital and analogue. In display's terminology, the digital display presents the information directly in numbers or digits. With the analogue display the humans have to interpret the information from the position of a pointer on a scale, from the shape, position or inclination of a picture on the screen, or from some other indication which is analogous to the real state of the functionable system [3].

One of the most common examples for digital and analogue displays, familiar to everyone, is in the wristwatch. The traditional analogue display represented by the circle with printed numbers where arms to point the hours, minute, and seconds. The other option is the digital wristwatch, where the time is presented by digits (numbers) in a Liquid Crystal Display (LCD) or similar. The selection between a digital or analogue wristwatch could be considered as a matter of personal preference and style. However, it could not be always said for the complex functionable systems, where numerous factors must be considered in order to select the one that best suits the requirements $[4,5]$.

To this paper, the VDDI are classified in the following three main groups:

Quantitative: This type of displays is a must when the actual values of the variable measured is required to be known with certain degree of precision. The quantitative VDDI, according to Sanders [6] can be of three types:

- $\quad$ Fixed scale with moving pointer (analogue)

- $\quad$ Moving scale with fixed pointer (analogue)

- Digital display, which according to Pankove [7], can be:

- $\quad$ Light-Emitting Diodes (LED),

- $\quad$ plasma, Liquid-Crystal Display (LCD),

- $\quad$ Electrochromic Displays (ECD)

- $\quad$ Electrophoretic Displays (EPID).

Qualitative: The qualitative types of VDDI are used when precision is not required. For example, the machine temperature in degrees centigrade, as the operator may simply need to know whether is hot, neutral, or cold, or whether is safe, dangerous or critical. This type of display is mostly analogue and used as a form of check reading. Kelly [5] indicates that qualitative displays usually represent a physical analogy between the display and that which it provides information about. If the spatial correspondence is such that the display resembles that which it represents, the display is said to be "pictorial".

Computerised VDDI: As Hancok [8] argues, the development of microchip technology, has led directly to more complex and interactive machines, complicating the problem of designing machines that are operable by human users. As systems get more complex, there are more cognitive tasks, more complex application and more interactive interfaces. Thus, the importance of the new concept of intelligent interfaces. Although is beyond the scope of this paper, it is important to be aware of the existence of this type of display. The computerised display can have numerous variants. Those used in industrial plants show digital, analogue, qualitative and quantitative information, if required, all of them simultaneously. The information can be presented, customised and can be changed at any moment to suit the needs of the user, all in one monochromatic or colour screen. This type of displays also offer the possibility of presenting the information in a representational o mimic form, with schematics and figures that intent to represent the parts of the system or variables that are being shown. Another advantage of this type of displays is that using "touch screen" capabilities is possible 
to access more detailed information of a particular item in a fully logical and intuitively sequence form for the operator. Even though this type of displays is most of the times related to automated type of process, it can also include the capability of taking control actions directly from the display. An example could be shutting down an equipment that shows nonacceptable levels of a relevant condition parameter.

\section{Uses of Visual Displays of Dynamic Information}

In order to select the adequate VDDI, it must be clearly defined what is the user expected to do with the display, this is, what is the intended use of the VDDI and what is the objective of using it. The selection of the VDDI should consider how the users will interact to achieve the desired requirement, observing their limitations.

According to James [2] the most important objectives of using VDDI, seen through the human perspective are as following:

- Improve decision-making (human nature is subjective).

- Improve understanding (gain insight of a problem or situation).

- Clarify complex relationships (relationships, conflicts, correlation, extrapolations, quick clearly and correctly comprehended).

- $\quad$ Reduce reaction times (detecting trends, conflicts and exceptions, planning for contingency).

- Improve co-ordination (between diverse operations)

- Improve availability of information.

- Improve control of the system.

- Simplify operations (simplicity, if equipment is difficult or inconvenient to use, it won't be used effectively)

- Provide flexibility (system able to adapt to changing requirements).

The selection process of the VDDI, will be briefly described below, in accordance to the MIRCE Science.

\section{Elements of selection process of VDDI type during the design}

Although every industry usually has some basic isolated guidelines for the selection of VDDI, particularly for systems that are designed in a recurrent manner, there is not a generic recipe for the selection process. Every case may have its own characteristics from operational, maintenance, logistics, etc. perspectives, in different environments, and all these characteristics can be related with ergonomic considerations, the possible combination of factors are almost infinite. Then, every situation must be evaluated with the people involved in operation and maintenance under intended circumstances, having human elements of functionable systems in mind is crucial for the selection of the VDDI.
From the information provided by Sanders [6] is possible to infer some aspects to be considered in the selection of the VDDI, these may include graphics, access, size, colors, arrangement, scales, environmental conditions and nature of function/process. These factors could influence the selection of the analogue or digital VDDI and are briefly discussed below.

\section{Graphics}

The types of questions that the Design Team should be focused on regarding the selection of VDDI are of the following nature:

- Does the user will require qualitative or quantitative information?

- $\quad$ Are symbols required?

- What is the nature of the process to be monitored?

- If a digital VDDI is considered what type is the best?

- What type of matrix shall be used?

- Does a mimic panel solve the situation from the user, operational and economical point of view?

- $\quad$ There are requirements of control associated with the displays to be used.

For example, in the case of VDDI used for cars is important to enable the driver to easily differentiate one display from another, like speedometer and rev. counter that are usually of the same size. This can be done using symbols associated with the VDDI and avoiding situations in which the driver may confuse two VDDI.

\section{Access and size}

Most of the VDDI are able to receive a remote signal, mechanical, electrical or digital from a sensing instrument, Hence, they don't have to be installed directly on the device whose parameter is being measured. The location (distance, viewing angle, etc.) of the operator in relation with the location of the VDDI is a factor to be considered when selecting between digital or analogue,, which in turns determines the diameter of the round analogue display or the size of the numbers in a digital one?. James [2] indicates that no matter how large displays get, people wish they were bigger. One cannot make the general statement that "the bigger the better", in fact, James indicates that a study compared a large purpose display for many people to view simultaneously with individual displays in front of each user. He concluded that there was some need for both. As Sanders [6] points out, to determine the size of the display, there are proven methods, including tables, depending on the reading distance and other factors.

\section{Colors and illumination}

Depending on the nature of the parameter to measure some colors are more convenient than others. There are some practices for the selection of colors associated to VDDI. Bailey [9] indicate some recommendations such red for abnormal/undesirable 
condition, yellow for warning, green for normal, etc. If the use of colors is required for an easier reading of the parameter measured, then strong consideration should be taken towards the use of analogue VDDI. The use of colors is particularly important in quantitative and qualitative VDDI, since they help to interpret quickly the functionability state of the parameter measured. The use of different colors in digital VDDI (blue, green, amber, red, etc.) can help to differentiate parameters, but they are not of much help.

The illumination of the VDDI is important, particularly if the system is intended to use at night or in places with poor illumination. Direct or indirect illumination shall be considered for both analogue and digital VDDI. The incandescent digital displays have an advantage in this situation since it does not require extra illumination at night, but if it is located outdoors, it does require adequate power during the day to overcome the brightness of the solar light in order to enable its reading.

\section{Scales}

The scale of the VDDI, especially in the analogue type of display should be carefully selected, not only foreseeing the expected range of operation, but also considering that the operator should easily read from it and differentiate from other displays. Some practices for the selection of scales are indicated by Bailey [9]. The Design team should consider the selection of VDDI that uses colors in their scales for the already discussed advantages. This is particularly important when selecting analogue qualitative type of VDDI, where the different ranges of operation could be identified using different colors. In digital VDDI, the scale (increments) represents the precision that the display must measure determined parameter. Most of the times, the process will dictate what is the required scale. For example, an aircraft digital altimeter measuring altitude in 5 [feet] increments could be acceptable but increments of 5 [gallons] in a digital meter of a petrol pump, would probably be not acceptable for the petrol station owner. Kelly [5] indicates some techniques for display scale determination and some recommended scales for various devices [10].

\section{Nature of function or process}

Depending on the nature of the process one type of display may have more advantages over others, again the important issue is to select the one that best suits the user, considering also related aspects as environment, maintenance, costs, etc. For example, analogue VDDI is an easy way of doing quick "check readings" whilst a digital VDDI could be a good choice for a parameter that need to be counted in a cumulative or historical way, such the odometer of a care. Nevertheless, the type of interaction user-display should be also considered: the length of the time the user will be interacting with the display. Is important to avoid that the users get tired of seeing a display not "eye-friendly".

\section{Arrangement}

It is critical to situate adequately the different types of displays of a system. The preference should be to group them in logical ways, according to sub-process, or criticality of the nature of the parameter. Kelly [11] indicates that the arrangement of the VDDI should be made according to the following priority:

- Function

- Importance

- Use sequence

- Frequency of use

The adequate location of the analogue VDDI enable the use of concepts such "positional concordance for the reading of multiple normal operating values" which is exposed by Sanders [6]. This concept can be useful when the user wants to verify the normal functioning of a system or part of it with a quick look at the set of VDDI. For example, if a certain number of analogue VDDIs are grouped together and are of similar sizes and the normal value of the parameter that each of the displays is measuring is positioned so that the arm of the display points in the same direction (say 12 o'clock in a round dial) then, instead of a series of random positioned pointers, the user could see, if everything is normal, a group of fairly aligned pointers, then any pointer out of alignment would be easily identified from the group. This type of arrangement is useful even for not so complex systems, such a automobile with a set of three VDDI.

\section{Environment}

Awareness of the environment where VDDI is going to be used is of vital importance, especially if it will be outdoors, exposed to weather conditions or extreme operational conditions. In that case it is crucial to assess will the operator be able to withstand the environmental conditions during its reading.

Also, it is important to assess will it be safe environment, and if not, how to provide protection for the user. At certain locations environmental conditions could affect users to make correct readings (illumination, heat, noise, vibration, wind), so the selection between digital or analogue VDDI became safety significant issues.

\section{The Final selection of the VDDI}

One of the main decisions that the design team has to make, is the selection of the VDDI, between digital and analogue. Today, most of new systems incorporate the use of chip technology to control their functions and performance. A direct way to communicate the information about their state is through digital output, their basic language. Also, the chip and digital display technology is becoming increasingly popular and more affordable, some digital displays are now cheaper than their analogue equivalents. As Osborne [3] argues, the experiments done by Murrell [10], tend to point that digital displays produces less reading errors and faster reading times (when a value is required) that their analogue counterparts. The digital clock can be read faster than the conventional clocks, with one tenth of the errors. From this affirmation, the impression may be gained, so far, that numerical information should always be 
presented digitally. Digital displays, however, have their weak side, particularly when the task requires some degree of "check reading", when the display is used as a predictor or when the rate of change of the information is fast.

The natural world where we live is mostly a "world of analogies". For thousands of years, humans have been used to see and understand all phenomena through analogies, seeing relative and gradual movements among parts. The digital presentation of information is entirely arbitrary and conceptual. This may be one of the main reasons why, humans may feel more comfortable reading analogue displays that enables them to use their intuition. The digital displays require an additional effort to actually "read" a value in a display and then find the meaning of its value against some referential scale that the user has to know, for the value to have some meaning. This is expressed by Kelly [11] when he points that "digital displays employ symbols such as numbers that can have no meaning to the person unfamiliar with the code or convention. One who is familiar with the code or convention, however, can interpret the information. In the other hand, with the analogue display, the user gets an instantaneous picture of what is going on with the variable, and the use of pictorial type analogue displays, the user requires minimum interpretation, as the nature of the analogy is obvious".

In comparing the advantages and disadvantages of digital and analogue VDDI, Osborne [3] made the conclusions that are presented below.

\section{Check reading}

Murrell [10] discuss a data from a survey of ships, which indicates that $18 \%$ of the dials of steam boilers were used for quantitative reading, whereas over $75 \%$ for some form of checking.

The digital displays lack of the perceptual information offered by the analogue type (for example the angle between a pointer and the "north") so the only way that the check reading can be done with digital is comparing numbers. For this reason, in certain cases, digital displays may increase both the time needed to read the instrument and the number of errors made during the task. This was demonstrated by Conell [12].

\section{Spatial clues for prediction}

"Speed and accuracy are only half of the problem. Very often the operator has to translate what he sees into appropriate action" [13]. For example, a digital clock may indicate that time is 11:40 a.m., an analogue clock indicating the same time enables quick spatial prediction, showing that there is 20 minutes left to reach 12 , with no further calculation.

\section{Blurring}

When the values to be read change too quickly, it may be difficult to read them from a digital display. In this case the spatial clues provided by the pointer in an analogue display may help the operator in making his reading.

From the previous analysis, it seems that there is not a simple answer when it comes to decide between a digital and analogue VDDI, there is not a recipe to follow. Every case must be evaluated considering its particular factors, some of them were discussed previously in this paper. Sometimes a digital VDDI will be the best solution for a particular situation, like recording quantitative information such the number of gallons of fuel pumped to a ship in a refinery pier. In other situation an analogue display will be the best solution, like when check reading is required using a manometer in a pressure vessel to determine if the pressure level is in a safe range. The final decision relays on the design group, that work together with user and maintenance personnel. This group will have to find what is the best type of display for every situation, is mainly an engineering decision. The merits of analogue and digital displays are summarized by Osborne [3] in (Table 1).

Table 1: Merits of analogue and digital displays in relation to intended function.

\begin{tabular}{|c|c|c|}
\hline Function & Analogue & Digital \\
\hline $\begin{array}{c}\text { Quantitative } \\
\text { reading }\end{array}$ & $\begin{array}{c}\text { Best is precise reading is not required, or if tasks contains predic- } \\
\text { tive or checking components. }\end{array}$ & $\begin{array}{c}\text { Best for accurate reading of slow changing values, poor if } \\
\text { task contains predictive or checking components. }\end{array}$ \\
\hline $\begin{array}{c}\text { Qualitative reading } \\
\text { Setting and track- } \\
\text { ing }\end{array}$ & Best for warnings, checking and prediction, useful to have visual \\
coded areas. & Poor \\
\hline
\end{tabular}

\section{Example if VDDI Selection}

To illustrate some considerations on the selection of VDDI, an example for a passenger car will be briefly considered.

The selection of VDDI for a car represents a situation where, from the human's point of view, there are some particular considerations:

- Most of the times, the user is not a specialised operator (has not received any training on the VDDI of the car) and may be or not familiarised with the automobile VDDI (if for example the user is renting a car).

- $\quad$ The user not only has to deal with the driving task which takes most of his attention, but also is responsible for reading and interpreting the parameters of the car through the VDDI at the same time.

During the driving, the user will have to check the values of the displays to assure the required functioning of the car. This situation 
represents a problem because he will have to take his sight out of the road while driving to read the displays (speed, petrol, oil pressure, temperature, voltage, amperage, etc.) just to assure that everything is running within the expected range/value. At a speed of $60 \mathrm{MPH}$ a car covers about 27 meters in one second. If the driver spends, say 3 seconds "reading" displays, the car would have run more than 80 meters with the driver not looking at the road. This is a potentially dangerous situation. If an obstacle suddenly appears in front of the car while the driver is not looking, even 1 second could make the difference between an accident and an evasive manoeuvre. For this reason, there is not enough time for the driver to read every gauge, instead he has to take short "looks" at instruments, he actually does "check reading". This is one of the main considerations that guide the selection of the VDDI in a car. Thus, the analogue VDDI should be the best suited for this task of check reading.

The car industry has almost 100 years of experience designing automobiles and selecting their VDDI. A quick research was done to find out the preference of car manufacturers in the use of VDDI, see (table 2) [14].

Table 2: Type of VDDI used by a random sample of automobiles.

\begin{tabular}{|c|l|}
\hline Make and Model & Type of VDDI \\
\hline Ford Escort & Analogue \\
\hline Citroen 2CV6 Special & Analogue \\
\hline Volvo 480 ES & Analogue \\
\hline Peugeot 205 GR & Analogue \\
\hline Fiat Uno 70S & Analogue \\
\hline Nissan Blue Bird & Analogue \\
\hline Toyota Corolla GL & Analogue \\
\hline Rover 218 SLD & Analogue \\
\hline BMW 325 tds & Analogue \\
\hline Mazda XEDOS9 & Analogue \\
\hline Rover 216 Gsi & Analogue \\
\hline Renault Laguna & Analogue \\
\hline
\end{tabular}

From the sample of 12 cars, Zavala [14] found that all of them use analogue type of VDDI for all dynamic parameters that require measurement, with the exception of odometers which were mechanical or electronic digital type of display. As a result of this short research it is evident that there is a preference for the use of analogue VDDI in cars. This is the expected result, since the design of the control panel of most cars is done for humans, which are an integral part of transportation work expected to be done by them, in accordance to MIRCE Science. When the user looks at an analogue VDDI a quick look is enough to locate the position of the arm of the display, and with the aid of a colour scale or qualitative indication, is possible to get an instant feeling of the parameter value. This is particularly important in parameters with high rate of change, like for example the tachometer. It would be very difficult to read values of RPM in a digital tachometer whilst changing gears, without taking the eyes out of the road for long periods of time (and still not been able to read a particular value due to "blurring"). The user, usually does not require to do any check reading on the odometer while driving, is mostly used for maintenance purposes, this is the reason why it does not matter if it is in a digital presentation.

The regular user finds easier to get information of the car parameters through the use of analogue VDDI. Also the fact that the driver only need approximate information for most of conditions of the car (with exemption of speed, which has to be controlled closely to avoid speeding ticket) encourages the use of qualitative analogue displays for parameters such as oil pressure, water temperature, voltage, amperage, etc.

\section{Quantitative Assessment of the Impact of VDDI on Work Done}

As of now, there is not a unique answer to the question which of which VDDI is better to use in a new system. The selection of the VDDI between digital and analogue is a tradeoff process that could be successfully supported by the quantitative evaluation of each design solution. That is possible by making use of MIRCE Functionability Equation [15]. It is able to consider each process during the life of a functionable system where VDDI are used and calculate the cumulative impact of all of them on the work done by the system and the cost of resources expected to be consumed.

\section{Conclusion}

Visual Displays of Dynamic Information (VDDI) represent the interface that machines use to communicate their dynamic functionability state to humans.

The main objective of this paper was to address the question of the selection between the two main type of VDDI available, digital or analogue, and identify what some of the most important factors are that may affect this selection. In that aid the paper briefly covered how the displays and VDDI are classified, their uses and objectives, the factors that could affect the selection process and finally a comparison between digital and analogue VDDI are made. As no definite answer was found in existing literature, the author 
introduced MIRCE Functionability Equation [15] as a method for quantitative assessment of the impact of VDDI on the human elements of functionable systems. Thus, the main conclusion of the paper is that for each feasible application of VDDI a quantitative assessment of the expected work and resources demanded has to be performed and the most compromising option selected, in accordance to the given criteria [16].

\section{Acknowledgement}

None.

\section{Conflicts of Interest}

No conflicts of interest.

\section{References}

1. Knezevic J (2017) The Origin of MIRCE Science, MIRCE Science, Exeter UK, pp. 232.

2. James H (1963) Electronic Information Display Systems, Cleaver-Home Press, London, pp. 6-18.

3. Osborne DJ (1982) Ergonomics at Work, John Willey \& Sons, Norwich, pp. 82-109

4. Knezevic J (1997) Systems Maintainability, pp 400, Chapman \& Hall, London, UK, 1997.
5. Singleton WT, Fox JG, Whitfield D (1971) International Ergonomics Association Simposium held in Amsterdam. Taylor and Francis Ltd, London, pp. 177-187.

6. Sanders MS, Mc Cormick J (1993) Human Factors in Engineering and Design, Mc. Graw - Hill, Singapore.

7. Pankove JI (1980) Display Devices, Springer-Velarg, Berlin.

8. Hancok PA, Chignell MH (1989) Intelligent Interfaces, North - Holland, New York.

9. Bailey RW (1982) Human Performing Engineering, Prentice - Hall, New Jersey, pp. 245-262.

10. Murrell KFH (1971) Ergonomics: Man, in His Working Environment, Chapman \& Hall, London, quoted by Osborne [3].

11. Kelly CR (1968) Manual and Automatic Control, John Willey \& Sons, New York, pp 91-105.

12. Conell (1948) Psychological Factors in Check Reading Single Instruments, USAF Air Material Command Memo Report No. MCREXD694-17A, quoted by Osborne [3].

13. Graham NE Human Factors in Equipment Design, Lewis, London, quoted by Osborne [3].

14. Zavala R (1995) Human Factors in Systems Design Selection of Adequate Visual Displays for Dynamic Information (VDDI), a research project for the master's degree in Reliability Engineering, MIRCE Centre, Exeter University, Exeter, UK.

15. Knezevic J (2014) MIRCE Functionability Equation, Int. Journal of Engineering Research and Applications, 4(8) pp 93-100. 